\title{
Prevalencia de vejiga hiperactiva en la Ciudad de México. Resultados de un análisis preliminar
}

\section{Prevalence of overactive bladder in Mexico City. Results of a preliminary analysis}

\author{
José Cruz-Ruíz, ${ }^{1}$ Viviana Farrera-González, ${ }^{1}$ Adrián Ransom-Rodríguez, ${ }^{1}$ Julio César Avalos-Jiménez, ${ }^{2}$ Martín \\ Landa-Soler, ${ }^{2}$ Adolfo González-Serrano, ${ }^{3}$ Roberto Cortez-Betancourt, ${ }^{3}$ Delfino de Jesús Marín-Acosta, ${ }^{4}$ Jesús \\ Torres-Aguilar, ${ }^{4}$ Javier A. Herrera-Muñoz, ${ }^{5}$ Mauricio Cantellano-Orozco, ${ }^{5}$ Melchor Castro-Marín, ${ }^{6}$ Nayelli Sosa- \\ Anau, ${ }^{7}$ Arturo García-Mora, ${ }^{1}$ Francisco Rodríguez-Covarrubias ${ }^{1}$
}

\begin{abstract}
Resumen
OBJETIVO: Determinar la prevalencia de vejiga hiperactiva y su repercusión (grado de afectación) en la calidad de vida de pacientes captados en cinco centros hospitalarios de la Ciudad de México.

MATERIALES Y MÉTODOS: Estudio descriptivo, transversal y observacional efectuado mediante la recolección prospectiva de expedientes provenientes de la consulta externa de Medicina general, Medicina familiar o Medicina interna de cinco centros hospitalarios de la Ciudad de México, entre enero y diciembre de 2017. Se solicitó a los participantes responder el cuestionario ICIQ-OAB (versión validada en español), en una sola ocasión y durante la misma visita. Los pacientes se dividieron en grupos etarios decenales a partir de 18 años. Para el análisis de los datos se utilizó estadística descriptiva.

RESULTADOS: Al momento del análisis intermedio, se contó con las encuestas de 352 participantes. El $72 \%(n=253)$ reportó urgencia urinaria, como síntoma cardinal de vejiga hiperactiva (78 vs $66 \%$ en mujeres y hombres, respectivamente, $\mathrm{p}<0.05$ ). Los síntomas moderados-severos se reportaron en $16 \%$ de los pacientes mayores de 57 años de edad. Los síntomas severos fueron más frecuentes en mujeres $(68 \%)$. El $31 \%$ de los casos refirió afectación moderada-severa en la calidad de vida. El principal síntoma concomitante fue nicturia $(81 \%)$; sin embargo, la incontinencia urinaria de urgencia provocó mayor molestia en los pacientes (32\%).

CONCLUSIÓN: La urgencia urinaria (síntoma cardinal de la vejiga hiperactiva) es una alteración con elevada prevalencia en la población de la Ciudad de México.

PALABRAS CLAVE: Síntomas de la vía urinaria inferior; vejiga hiperactiva; calidad de vida.

\section{Abstract}

OBJECTIVE: To determine the prevalence of overactive bladder and the degree of impact it has on the quality of life of patients recruited from five hospital centers in Mexico City. MATERIALS AND METHODS: A descriptive, observational, cross-sectional study was conducted through the prospective collection of patient data from the General Medicine, Family Medicine, or Internal Medicine outpatient consultation at five hospital centers in Mexico City, within the time frame of January and December 2017. Participants were asked to fill out the validated Spanish version of the ICIQ-OAB questionnaire at that same consultation, one time only. The patients were divided into ten-year age groups starting at 18 years of age. The data were analyzed through descriptive statistics. RESULTS: There were questionnaires from 352 participants at the time of the intermediate analysis. A total of $72 \%(n=253)$ reported urgency as the key symptom of overactive bladder (78 vs $66 \%$ in women and men, respectively, $\mathrm{p}<0.05$ ). Moderate-severe symptoms were reported in $16 \%$ of the patients above the age of 57 years. Severe symptoms were more frequent in women $(68 \%)$. A total of $31 \%$ of the patients described a
\end{abstract}

${ }^{1}$ Departamento de Urología, Instituto Nacional de Ciencias Médicas y Nutrición Salvador Zubirán, Ciudad de México. ${ }^{2}$ Departamento de Urología, Hospital Regional Lic. Adolfo López Mateos, ISSSTE, Ciudad de México.

${ }^{3}$ Departamento de Urología, Centro Médico Nacional 20 de Noviembre, ISSSTE, Ciudad de México.

${ }^{4}$ Departamento de Urología, Hospital Juárez de México, Ciudad de México.

${ }^{5}$ Departamento de Urología, Hospital General Dr. Manuel Gea González, Ciudad de México.

${ }^{6}$ Departamento de Urología, Hospital Central Militar, Ciudad de México.

${ }^{7}$ ASEMEI consultores, Ciudad de México.

Recibido: marzo 2018

Aceptado: mayo 2018

Correspondencia

Francisco Rodríguez Covarrubias

frodriguez.covarrubias@gmail.com

Este artículo debe citarse como Cruz-Ruíz J, Farrera-González V, Ransom-Rodríguez A, Avalos-Jiménez JC, Landa-Soler M, González-Serrano A y col. Prevalencia de vejiga hiperactiva en la Ciudad de México. Resultados de un análisis preliminar. Rev Mex Urol. 2018 mayo-junio;78(3):189-196. DOI:https://doi.org/10.24245/revmexurol.v78i3.2109 
moderate-severe impact on quality of life. The main concomitant symptom was nocturia $(81 \%)$ but urge incontinence was the most bothersome symptom for patients $(32 \%)$.

CONCLUSION: The prevalence of urgency (the cardinal symptom of overactive bladder) was high in a population in Mexico City.

KEYWORDS: Lower urinary tract symptoms; Overactive bladder; Quality of life

\section{ANTECEDENTES}

La expresión "síntomas de la vía urinaria inferior" se introdujo en 1994 y se refiere a las diferentes manifestaciones que afectan a la vejiga, uretra, y en hombres la próstata; se clasifican en síntomas de almacenamiento, vaciamiento y posmiccionales, ${ }^{1,2}$ y pueden expresarse en pacientes con enfermedades de la vía urinaria inferior como: crecimiento prostático, vejiga hiperactiva, alteraciones en la uretra o los riñones. Se estima que $76 \%$ de los adultos ha experimentado, al menos, un episodio de síntomas de la vía urinaria inferior. $^{3}$ Diversos estudios epidemiológicos señalan que estos síntomas se incrementan con la edad; sin embargo, los de almacenamiento son los más prevalentes e interfieren de manera directa en la calidad de vida. ${ }^{3-5}$ La vejiga hiperactiva es la causa principal de los síntomas urinarios de almacenamiento y se define, de acuerdo con la Sociedad Internacional de Continencia (ICS, por sus siglas en inglés), como una alteración caracterizada por urgencia urinaria, habitualmente con frecuencia urinaria y nicturia, con o sin incontinencia urinaria de urgencia, sin infección u otra complicación aparente. ${ }^{2}$ Recientemente, con base en las definiciones estandarizadas de la ICS-2002, se ha hecho un esfuerzo importante para efectuar estudios epidemiológicos que permitan estimar la prevalencia de vejiga hiperactiva en diferentes países de Europa, Norteamérica y Asia, ${ }^{3,4,6}$ mediante la evaluación de la urgencia urinaria como síntoma cardinal. Sin embargo, las cifras varían ampliamente, según la población estudiada y los métodos de recolección de datos (cuestionarios autoadministrados), con una estimación global de $10.7 \%{ }^{7}$
Todos los estudios coinciden en que la prevalencia de urgencia urinaria y vejiga hiperactiva se incrementa con la edad. ${ }^{4}$ De igual forma, se ha demostrado que la coexistencia y gravedad de los síntomas puede repercutir negativamente en la calidad de vida del paciente, ya sea por trastornos del sueño, ansiedad, depresión y deficiente desempeño laboral. ${ }^{8,9}$ Por esta razón es necesario contar con métodos diagnósticos que proporcionen una evaluación clínica precisa de los síntomas disfuncionales y su efecto en la calidad de vida. El proyecto modular de cuestionarios del ICIQ (International Consultation on Incontinence modular Questionnaire project) es uno los más recomendados actualmente para tal propósito, ${ }^{10,11}$ pues contiene propiedades psicométricas firmes y de autocumplimentación por los pacientes (grado de recomendación A) para evaluar los síntomas de la vía urinaria inferior de manera exhaustiva, determinando la manifestación, gravedad y efecto individual que supone cada síntoma en la calidad de vida, todo ello de acuerdo con las definiciones actuales del ICS y validado en idioma español. ${ }^{12,13}$

De acuerdo con la bibliografía consultada, la información acerca de la prevalencia de vejiga hiperactiva en México es limitada. Pocos trabajos se basan en la definición actual del ICS; por tanto, el objetivo de este estudio consistió en determinar la prevalencia de vejiga hiperactiva y su repercusión (grado de afectación) en la calidad de vida de la población mexicana, de acuerdo con las definiciones estandarizadas de la enfermedad. 


\section{MATERIALES Y MÉTODOS}

Estudio descriptivo, transversal y observacional efectuado mediante la recolección prospectiva de datos de hombres y mujeres mayores de 18 años de edad, provenientes de la consulta externa de Medicina general, Medicina familiar o Medicina interna de cinco centros hospitalarios de la Ciudad de México, entre enero y diciembre de 2017. Con la finalidad de reducir los sesgos de selección, se excluyeron los pacientes con infección de vías urinarias, con sonda urinaria o quienes se atendieron en la consulta externa de Urología y que no contestaron los cuestionarios en su totalidad.

Como parte de la evaluación se solicitó a los participantes responder de manera autoadministrada el cuestionario ICIQ-OAB (versión validada en español), ${ }^{11}$ en una sola ocasión y durante la misma visita. Para fines del análisis, los pacientes se dividieron en grupos etarios decenales a partir de 18 años. Los procedimientos, las definiciones y unidades coincidieron con la terminología y los conceptos recomendados por la ICS. ${ }^{1,2}$ Este estudio contó con la aprobación del Comité de Ética en Investigación del Instituto Nacional de Ciencias Médicas y Nutrición Salvador Zubirán (Referencia 2099).

\section{Cuestionario}

El cuestionario ICIQ-OAB comprende 4 ítems, con una escala Likert de 5 alternativas según la gravedad de los síntomas $(0=$ nunca, $1=$ algunas veces, 2 = bastantes veces, 3 = la mayor parte de las veces, 4 = siempre). Cada pregunta evalúa síntomas de vejiga urinaria y su repercusión en la calidad de vida. Los síntomas evaluados incluyen: nicturia, urgencia urinaria, frecuencia diurna e incontinencia urinaria de urgencia. La repercusión en la calidad de vida se evalúa con una pregunta para cada síntoma mediante una escala numérica de 0 (no me afecta nada) a 10 (me afecta mucho). ${ }^{11}$ Para fines del estudio consideramos como caso positivo de vejiga hiperactiva a los pacientes con el síntoma cardinal de urgencia presente. La gravedad de los síntomas asociados con vejiga hiperactiva se reportan con las siguientes equivalencias de la escala Likert: ocurre "algunas veces" = grado leve; ocurre "bastantes veces" = grado moderado; ocurre "la mayor parte de las veces" o "siempre" = grado severo. Para el puntaje de "afectación en la calidad de vida", según el síntoma evaluado, generamos tres categorías: 0 a 3 puntos = afectación nula o mínima, 4 a 6 puntos = afectación moderada y 7 a 10 puntos = afectación severa.

De acuerdo con la prevalencia de vejiga hiperactiva ( $11 \%$ ) reportada en la bibliografía, ${ }^{7}$ se calculó el tamaño de la muestra con la fórmula para estudios de prevalencia para variables expresadas en proporciones, con intervalo de confianza (IC95\%) y poder de $95 \%$, con lo que se obtuvo una proporción total de 798 participantes. Se consideró efectuar un análisis intermedio cuyos resultados aparecen más adelante.

\section{Análisis estadístico}

Se utilizó estadística descriptiva, mediante medidas de tendencia central (media, mediana, desviación estándar o rango intercuartil) para variables numéricas, y frecuencias y proporciones para variables categóricas. Los datos se analizaron con el programa SPSS v.22 (IBM Corporation, USA).

\section{RESULTADOS}

\section{Prevalencia de vejiga hiperactiva en la población general}

Al momento del análisis intermedio se contó con las encuestas de 352 participantes (50\% mujeres), con edad promedio de 51 años (límites de 18-85 años). De acuerdo con la definición 
estandarizada del ICS-2002: $72 \%(n=253)$ de la población encuestada tenía urgencia urinaria, como síntoma cardinal de vejiga hiperactiva, lo que representó una prevalencia de $78 \%$ ( $\mathrm{n}=$ $137)$ en mujeres y $66 \%(n=116)$ en hombres $(p<0.05)$. Estos 253 individuos fueron el centro de nuestro análisis, con edad promedio de 52 años. El análisis por grupos de edad demostró que $56 \%$ eran menores de 57 años, aunque el grupo con mayor proporción de casos fue el de 57 a 66 años (23\%) Figura 1.

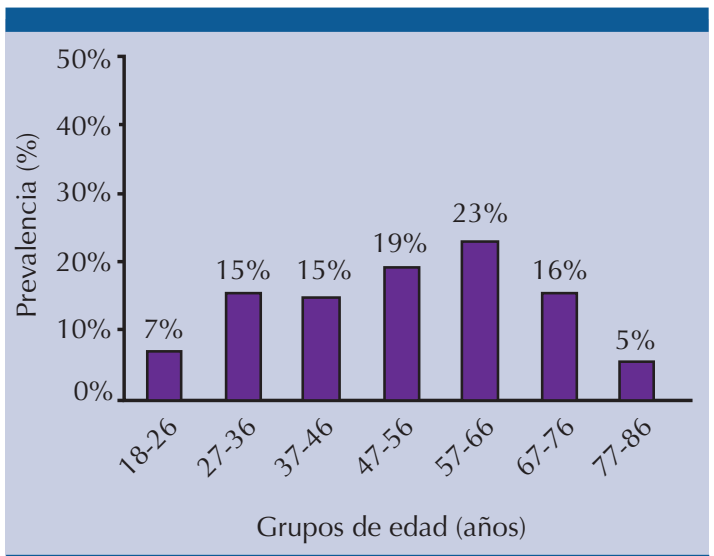

Figura 1. Pacientes con vejiga hiperactiva por grupos de edad (decena) $(n=253)$.

\section{Gravedad de los síntomas y afectación en la calidad de vida}

El 16\% de los pacientes con urgencia urinaria reportó síntomas moderados (6\%) a severos $(10 \%)$, con incremento en la gravedad de los síntomas a mayor edad. En general, los sujetos de entre 57 y 66 años reportaron mayor prevalencia de síntomas severos por vejiga hiperactiva ( $16 \%)$; sin embargo, se observó un incremento de síntomas moderados-severos a partir de los 57 años de edad (Figura 2a). Los síntomas de vejiga hiperactiva severos fueron más frecuentes en mujeres que en hombres (68 vs 32\%, respectivamente); por su parte, los hombres representaron $60 \%$ de la población con síntomas moderados. Los síntomas leves fueron similares en ambos sexos (mujeres $53 \%$ y hombres $47 \%$ ).

Después de analizar la proporción de pacientes con criterios de vejiga hiperactiva con afectación en la calidad de vida, se observó que 31\% refirió afectación moderada (22\%) a severa $(9 \%)$. Con base en este resultado, la prueba $U$ de Mann Whitney reportó relación estadísticamente significativa entre el grado de afectación en la calidad de vida y vejiga hiperactiva (IC95\%, $z=-5.740, p<0.05)$. No es sorprendente que conforme aumenta la gravedad de los síntomas de vejiga hiperactiva mayor es el deterioro en la calidad de vida. Cuando los síntomas son "leves", sólo 3\% de los pacientes reporta afectación "severa" en la calidad de vida y cuando los síntomas se manifiestan "la mayor parte de las veces" o "siempre" (severos), la calidad de vida se afecta de forma "severa" en la mayoría de los casos (52\%) (Figura 2b).

\section{Combinación de síntomas de vejiga hiperactiva}

Al evaluar los cuatro síntomas que definen los criterios diagnósticos de vejiga hiperactiva (urgencia urinaria, incontinencia urinaria de urgencia, frecuencia y nicturia), 92\% tuvo más de un síntoma, además de urgencia. La nicturia fue el síntoma más frecuente; $81 \%$ reportó al menos un episodio de nicturia, seguido de $52 \%$ con incontinencia urinaria de urgencia y $37 \%$ con frecuencia urinaria como síntoma concomitante. La incontinencia urinaria de urgencia fue el síntoma más molesto: $32 \%$ de los casos reportó afectación moderada (17\%) a severa (15\%) en la calidad de vida (Figura 3). El 19\% de los pacientes manifestó los 4 síntomas que definen el trastorno de vejiga hiperactiva (Cuadro 1).

\section{DISCUSIÓN}

La prevalencia de síntomas compatibles con vejiga hiperactiva en la Ciudad de México es alta (78 vs 66\% en mujeres y hombres, respecti- 


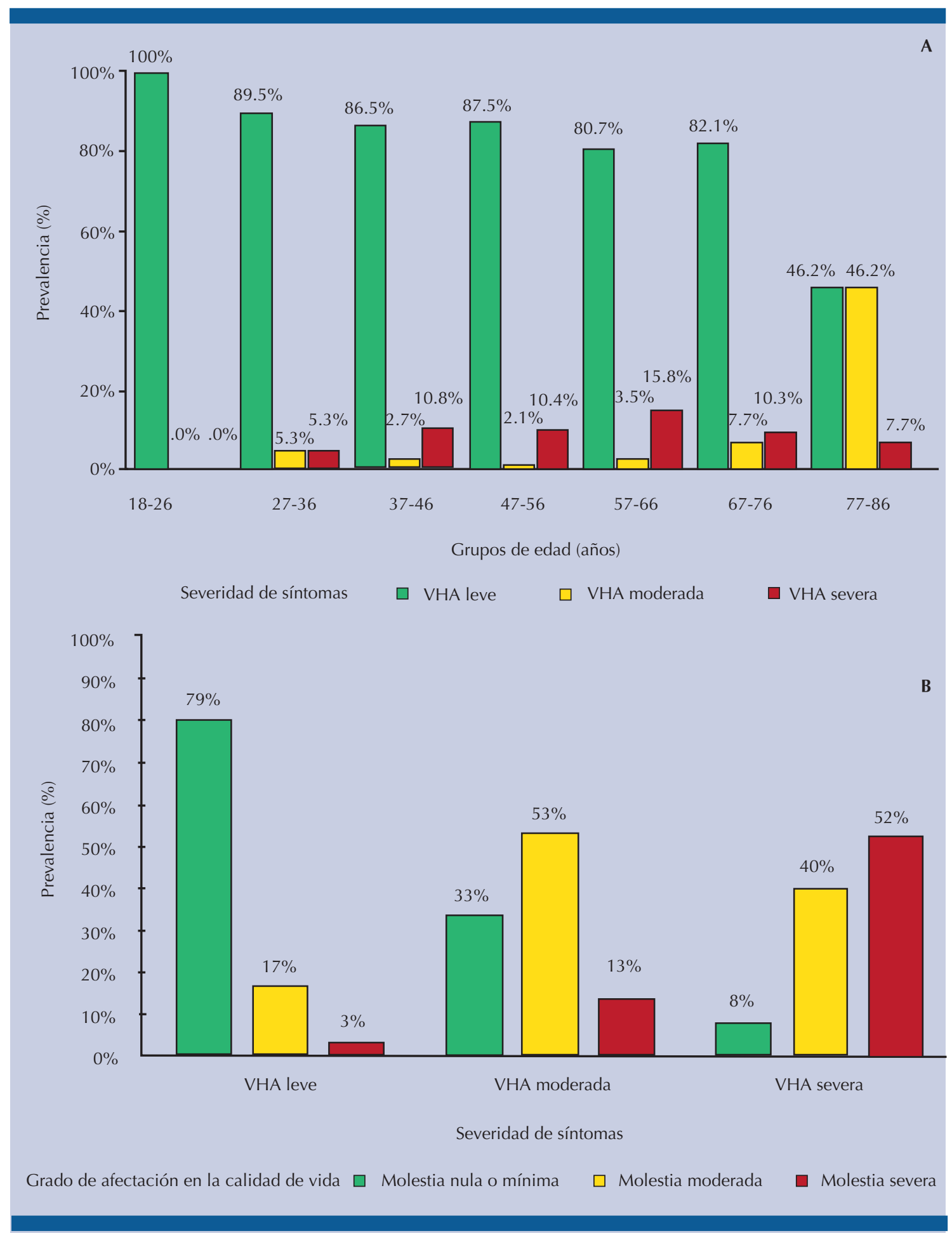

Figura 2. Estimación de la gravedad de los síntomas de vejiga hiperactiva (A) y afectación en la calidad de vida (B). VHA: vejiga hiperactiva. 


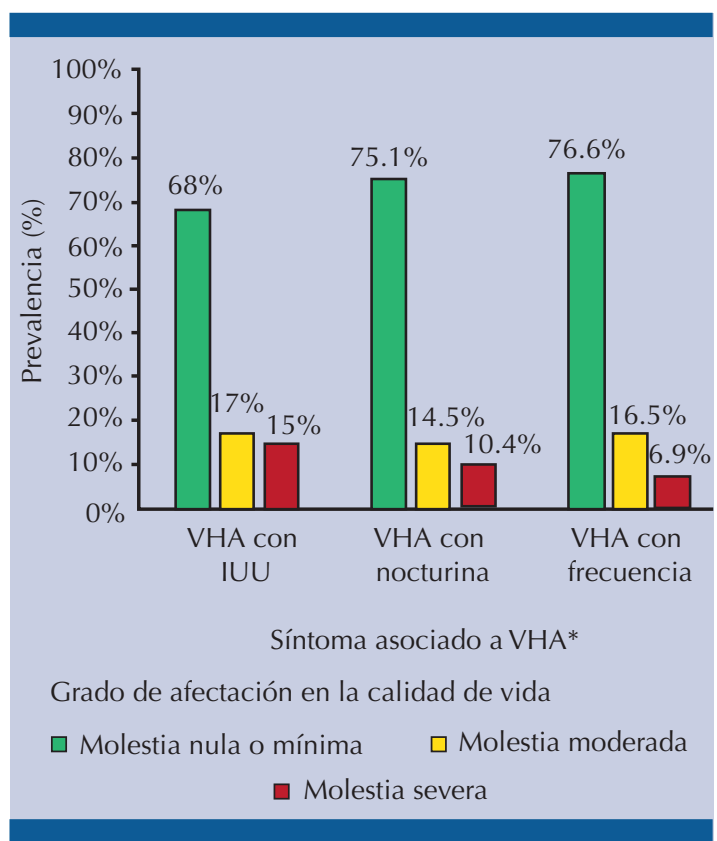

Figura 3. Pacientes con afectación en la calidad de vida (nula o mínima, moderada o severa) según el síntoma relacionado con vejiga hiperactiva.

VHA: vejiga hiperactiva; IUU: incontinencia urinaria de urgencia.

Cuadro 1. Síntomas de vejiga hiperactiva según su combinación $(n=249)$

\begin{tabular}{l|c|}
\hline Síntomas & $\%$ \\
\hline Urgencia & $8 \%$ \\
\hline Urgencia y frecuencia & $2 \%$ \\
$\begin{array}{l}\text { Urgencia e incontinencia urinaria } \\
\text { de urgencia }\end{array}$ & $6 \%$ \\
Urgencia y nicturia & $24 \%$ \\
\hline $\begin{array}{l}\text { Urgencia, frecuencia e incontinencia } \\
\text { urinaria de urgencia }\end{array}$ & $3 \%$ \\
$\begin{array}{l}\text { Urgencia, frecuencia y nicturia } \\
\text { Urgencia, incontinencia urinaria de } \\
\text { urgencia y nicturia }\end{array}$ & $14 \%$ \\
$\begin{array}{l}\text { Urgencia, frecuencia, incontinencia } \\
\text { urinaria de urgencia y nicturia }\end{array}$ & $24 \%$ \\
\hline $\begin{array}{l}\text { Urgencia y nicturia* } \\
\text { Urgencia e incontinencia urinaria de } \\
\text { urgencia* }\end{array}$ & $19 \%$ \\
\hline $\begin{array}{l}\text { Urgencia y frecuencia* } \\
\text { *Síntoma relacionado, solo o en combinación. } \\
* * \text { Prevalencia acumulada. }\end{array}$ & $81 \% * *$ \\
\hline
\end{tabular}

vamente), incluso mayor a la descrita en estudios internacionales, como el EPIC (mujeres 10.8\% y hombres $12.8 \%)^{4}$ y epiLUTS (mujeres $35.7 \%$ y hombres $22.4 \%)^{3}$ y la investigación de Castro y su grupo (mujeres $17.4 \%$, hombres $19.2 \%$ ). ${ }^{14}$ Estas diferencias pueden deberse a la disparidad étnica, pues recientemente se describió que la población hispana tiene mayor riesgo de padecer síntomas moderados o severos de la vía urinaria inferior. ${ }^{15}$ Las principales causas pueden relacionarse con factores orgánicos, estilo de vida, medioambientales o culturales.

Aunque las cifras pueden variar entre las distintas poblaciones, nuestros resultados coinciden en que la prevalencia y gravedad de los síntomas se incrementan con la edad, con mayor proporción de síntomas severos en mujeres y en los pacientes mayores de 57 años de edad. 8,16

El estudio de García-Sánchez y sus colaboradores ${ }^{1,7}$ efectuado con 171 médicos generales, reportó una prevalencia menor de vejiga hiperactiva (19.2 vs $16.3 \%$ en mujeres y hombres, respectivamente); no obstante, es complicado realizar una comparación directa con nuestros resultados, porque utilizaron definiciones y cuestionarios distintos.

La principal virtud del cuestionario modular ICIQ-OAB es que permite llevar a cabo una evaluación exhaustiva e individual de la gravedad de los síntomas y afectación en la calidad de vida del paciente, ${ }^{18}$ que es una característica de la que carecen otros cuestionarios, como el OAB-V8.

Similar a lo reportado por Agarwal y su equipo de trabajo, ${ }^{19}$ el síntoma relacionado con mayor prevalencia de urgencia urinaria fue la nicturia; sin embargo, el que provocó mayor afectación en la calidad de vida fue la incontinencia urinaria de urgencia. Este hallazgo coincide y fortalece la evidencia a favor de estudios que establecen una fuerte relación entre incontinencia urinaria de urgencia y deterioro en la calidad de vida, debido 
a su asociación con ansiedad y depresión, 9,19,20 incremento de costos (antimuscarínicos y protectores íntimos para la incontinencia urinaria) e interferencia con el trabajo y actividades de la vida diaria. ${ }^{6}$ Desde el punto de vista poblacional, este estudio demuestra la alta prevalencia de vejiga hiperactiva en la Ciudad de México. Se estima que tres cuartas partes de las mujeres y dos terceras partes de los hombres manifiesta síntomas compatibles con la enfermedad, 50\% padece incontinencia urinaria de urgencia y una tercera parte tiene calidad de vida afectada en grado moderado-severo. Por ello, se recomienda utilizar cuestionarios estandarizados y validados en idioma español, que no subestimen los síntomas de almacenamiento ni los de incontinencia, además de permitir una adecuada evaluación de la calidad de vida del paciente.

Entre las limitaciones de nuestro estudio se encuentra la aplicación de cuestionarios de autorrespuesta para evaluar los síntomas de la vía urinaria inferior, como herramienta para establecer el diagnóstico de vejiga hiperactiva. El adecuado diagnóstico de esta alteración se basa en la rigurosa evaluación clínica y funcional de dichos síntomas, que en casos complejos requiere el apoyo de estudios urodinámicos; sin embargo, este tipo de evaluación no puede llevarse a cabo en un estudio epidemiológico de tal escala. Otra limitación es que los cuestionarios no distinguen las manifestaciones relacionadas con otras alteraciones de la vía urinaria inferior, solo las de vejiga urinaria; por tanto, la prevalencia estimada, basada sólo en la coexistencia de urgencia urinaria puede sobrestimar el diagnóstico. Este problema es inherente al resto de los estudios epidemiológicos y representa un sesgo común que debe considerarse al interpretar y comparar los datos.

Reconocemos que nuestras cifras están limitadas a la población de la Ciudad de México y no necesariamente se generalizan a otras localidades del país. No obstante, representa un esfuerzo multicéntrico apoyado por la Sociedad Mexicana de Urología, con la mayor serie de pacientes con vejiga hiperactiva en México. Aun así, se requieren estudios con mayor número de pacientes y con alcance nacional, que permitan estimar de mejor manera la prevalencia de vejiga hiperactiva.

Estos datos tienen importantes implicaciones para la práctica clínica y forman parte de un esfuerzo continuo por conocer la prevalencia y repercusión de los síntomas de la vía urinaria inferior en la población mexicana, que inició algunos años atrás con el estudio MexiLUTS, ${ }^{21}$ y tiene como objetivo generar conocimientos en nuestra población.

\section{CONCLUSIÓN}

De acuerdo con la definición actual de la Sociedad Internacional de Continencia (ICS), la prevalencia de síntomas compatibles con vejiga hiperactiva en la Ciudad de México es elevada, incluso aumenta con la edad, y afecta la calidad de vida de la mayoría de los pacientes. Aunque la nicturia es el síntoma más frecuente, la incontinencia urinaria de urgencia provoca mayor deterioro en la calidad de vida. La proporción de pacientes con urgencia urinaria fue mayor a la reportada en otros estudios; sin embargo, se trata de un análisis intermedio, por lo que los resultados no pueden generalizarse.

\section{Agradecimientos}

Este trabajo se realizó con el apoyo del Consejo Directivo de la Sociedad Mexicana de Urología Colegio de Profesionistas, A.C. en su gestión 2016-2017.

\section{REFERENCIAS}

1. Abrams P, Cardozo L, Fall M, et al. The standardisation of terminology in lower urinary tract function: Report from the standardisation sub-committee of the International Continence Society. Urology 2003;61(1):37-49. doi:10.1016/S0090-4295(02)02243-4.

2. Haylen $B T$, de Ridder D, Freeman RM, et al. An international Urogynecological Association (IUGA)/International 
Continence Society (ICS) joint report on the terminology for female pelvic floor dysfunction. Neurourol Urodyn 2010;29(1):4-20. doi:10.1002/nau.20798.

3. Coyne KS, Sexton CC, Thompson CL, et al. The prevalence of lower urinary tract symptoms (LUTS) in the USA, the UK and Sweden: Results from the epidemiology of LUTS (EpiLUTS) study. BJU Int 2009;104(3):352-360. doi:10.1111/j.1464410X.2009.08427.x.

4. Irwin DE, Milsom I, Hunskaar S, et al. Population-based survey of urinary incontinence, overactive bladder, and other lower urinary tract symptoms in five countries: results of the EPIC study. Eur Urol 2006;50(6):1306-1315. doi:10.1016/j.eururo.2006.09.019.

5. McVary KT, Peterson A, Donatucci CF, et al. Use of Structural Equation Modeling to Demonstrate the Differential Impact of Storage and Voiding Lower Urinary Tract Symptoms on Symptom Bother and Quality of Life during Treatment for Lower Urinary Tract Symptoms Associated with Benign Prostatic Hyperplasia. J Urol 2016;196(3):824-830. doi:10.1016/j.juro.2016.04.062.

6. Lee KS, Choo MS, Seo JT, et al. Impact of overactive bladder on quality of life and resource use: results from Korean Burden of Incontinence Study (KOBIS). Health Qual Life Outcomes 2015;13(1):89. doi:10.1186/s12955-015-0274-9.

7. Irwin DE, Kopp ZS, Agatep B, Milsom I, Abrams P. Worldwide prevalence estimates of lower urinary tract symptoms, overactive bladder, urinary incontinence and bladder outlet obstruction. BJU Int 2011;108(7):1132-1138. doi:10.1111/j.1464-410X.2010.09993.x.

8. Irwin DE, Abrams P, Milsom I, Kopp Z, Reilly K. Understanding the elements of overactive bladder: Questions raised by the EPIC study. BJU Int 2008;101(11):1381-1387. doi:10.1111/j.1464-410X.2008.07573.x.

9. Coyne KS, Sexton CC, Kopp ZS, Ebel-Bitoun C, Milsom I, Chapple C. The impact of overactive bladder on mental health, work productivity and health-related quality of life in the UK and Sweden: Results from EpiLUTS. BJU Int 2011;108(9):1459-1471. doi:10.1111/j.1464410X.2010.10013.x.

10. Hashim $H$, Abrams P. Overactive bladder: an update. Curr Opin Urol 2007;17(4):231-236. doi:10.1097/ MOU.0b013e32819ed7f9.

11. Abrams P, Avery K, Gardener N, Donovan J. The International Consultation On Incontinence Modular Questionnaire: www.iciq.net. J Urol 2006;175(3):1063-1066. doi:10.1016/ S0022-5347(05)00348-4.
12. Castro-Díaz DM, Esteban-Fuertes M, Salinas-Casado J, et al. Evaluación de las propiedades psicométricas de la versión en español del cuestionario ICIQ-Male Lower Urinary Tract Symptoms (ICIQ-MLUTS) en España. Actas Urol Esp 2014;38(2):71-77. doi:10.1016/j.acuro.2013.07.005.

13. Espuña-Pons M, Rebollo-Álvarez P, Puig-Clota M. Validación de la versión española del International Consultation on Incontinence Questionnaire-Short Form. Un cuestionario para evaluar la incontinencia urinaria. Med Clin (Barc) 2004;122(8):288-292. doi:10.1157/13058677.

14. Castro D, Espuña M, Prieto M, Badia X. Prevalencia de vejiga hiperactiva en españa : estudio poblacional. Arch Esp Urol 2005;58(2):131-138.

15. Van Den Eeden SK, Shan J, Jacobsen SJ, et al. Evaluating racial/ethnic disparities in lower urinary tract symptoms in men. J Urol 2012;187(1):185-189. doi:10.1016/j. juro.2011.09.043.

16. Coyne KS, Sexton CC, Vats V, Thompson C, Kopp ZS, Milsom I. National community prevalence of overactive bladder in the United States stratified by sex and age. Urology 2011;77(5):1081-1087. doi:10.1016/j.urology.2010.08.039.

17. García-Sánchez D, Gutiérrez-González A, Pérez-Ortega R, Gastelum-Félix LA, Reyes-Gutiérrez M. Frecuencia de la vejiga hiperactiva en médicos generales. Rev Mex Urol 2014;74(2):84-87. doi:10.1016/S2007-4085(15)30017-3.

18. Shy M, Fletcher SG. Objective Evaluation of overactive bladder: which surveys should i use? Curr Bladder Dysfunct Rep 2013;8(1):45-50. doi:10.1007/s11884-012-0167-2.

19. Agarwal A, Eryuzlu LN, Cartwright R, et al. What is the most bothersome lower urinary tract symptom? Individual- and population-level perspectives for both men and women. Eur Urol 2014;65(6):1211-1217. doi:10.1016/j. eururo.2014.01.019.

20. Vaughan $\mathrm{CP}$, Johnson TM, Ala-Lipasti MA, et al. The prevalence of clinically meaningful overactive bladder: Bother and quality of life results from the population-based FINNO study. Eur Urol 2011;59(4):629-636. doi:10.1016/j. eururo.2011.01.031.

21. Gonzalez-Sanchez B, Cendejas-Gomez J, Alejandro RiveraRamirez J, et al. The correlation between lower urinary tract symptoms (LUTS) and erectile dysfunction (ED): results from a survey in males from Mexico City (MexiLUTS). World J Urol 2016;34(7):979-983. doi:10.1007/s00345015-1703-4. 